\title{
Analyzing Reception, Transformation and Problem Solving Competences in Professional Mathematics of University Students by Using GRA
}

\author{
Hsiu-Lan Ma, Der-Bang Wu, and Yu-Kuang Cheng
}

\begin{abstract}
This study analyzed the reception, transformation and problem solving ability of professional mathematics of university students of technology by using Grey Relational Analysis (GRA). In the nine-year Standards of mathematics curriculum published in $\mathbf{2 0 0 8}$, there is an important topic called "Connection." The first part of the "Connection" is called "reception." The second part of the "Connection" is called "transformation," "problem solving" followed. Current results of this study show that the problem concerned unemployment rate is the most difficulty among 10 problems, followed by the problem concerned sales profit.
\end{abstract}

Index Terms-Grey relational analysis (GRA), reception, transformation and problem solving, professional mathematics, mathematics, knowledge.

\section{INTRODUCTION}

The Grey Relational Analysis (GRA) is widely used in many fields, such as education, decision-making in economics, marketing research, medicine, computer science, system modeling, social science, chemistry, management, etc. Therefore, we use GRA as a new method to analyze the connect topic of university students.

We live in a mathematical world [1]. Whenever we decide on a purchase, choose an insurance or health plan, or use a spreadsheet, we rely on mathematical understanding [1]. While mathematics is an integrated arena of study, often students view concepts as disjointed chunks of information with little connection or relation [1]. According to National Council of Teachers of Mathematics (NCTM) [1], students from prekindergarten through grade 12 should see and experience the rich interplay among mathematical topics, see and experience this interconnectedness between mathematics and other domains, and also be able to connect mathematics to their own interests [1]. Viewing mathematics as a whole, such as web of linked concepts, will help students to learn that mathematics is not a set of isolated skills and arbitrary rules [1]. "Connection" is the term used in the national mathematics

Manuscript received October 29, 2014; revised January 7, 2015. This work was supported in part by the Taiwan Minister of Science and Technology (MOST) under Grant NSC 100-2511-S-275 -001-MY3 (Opinions expressed do not necessarily reflect the views of MOST).

H. L. Ma is with the Department of Business Administration, Ling Tung University, Taichung 40852, Taiwan (e-mail: hlma@ hotmail.com.tw).

D. B. Wu is with the Graduate Institute of Mathematics Education, National Taichung University, Taichung 40306, Taiwan (e-mail: wudb@ hotmail.com).

Y. K. Cheng is with the Department of English, National Taichung University, Taichung 40306, Taiwan (e-mail: mike8826us@yahoo.com). curriculum of Taiwan for addressing the issue of the relationships between mathematics and the other subjects.

In the nine-year mathematics curriculum of 2008 for primary and junior-high school [2], there is a topic called "Connection." The first part of the "Connection" is called "reception," which includes the following competences that students need to possess [2].

C-R-01 Can aware the daily contexts that are related to mathematics.

C-R-02 Can aware the Connect between mathematics and the other domains.

C-R-03 Can understand that mathematics can be applied to natural or social sciences.

C-R-04 Can provide concrete examples of how mathematics promote human cultural development.

The second part of the "Connection" is called "transformation," "problem solving" followed.

In the present high-school curriculum, one of the goals of mathematical curriculum is to cultivate student necessary mathematics knowledge and skills to improve daily practices and to learn related school subjects. One of the core competences of mathematics curriculum is "connection": To integrate internal mathematical knowledge and to connect to the concrete world [3]-[9].

Therefore, this paper aims to analyze the first 3 steps of connect [reception, transformation, and problem solving ability] of professional mathematics of university students by using Grey Relational Analysis (GRA).

\section{Methods AND PROCEDURES}

\section{A. Participants}

In this study, a questionnaire of 10 Problems was conducted and 207 students of University of Technology in Taiwan were asked to respond.

\section{B. Instrument}

This study uses the Ma-Wu's Test of Competences in Professional Mathematics of University Students (MWTCPM), which was specifically designed for this project, due to no suitable Chinese instruments available [10]-[15]. There are 10 multiple-choice problems. Each problem contained 4 sub-problems which collated the connection's 4 sub-steps: "reception", "transformation and problem solving", "communication", as well as "evaluation". The score for each sub-problem is one point. So, there are 4 points for each problem and totally 40 points in each test. However, in this paper, only the scores on the first three sub-steps are 
analyzed.

\section{GREY RELATIONAL ANALYSIS}

The grey relational analysis is depicted in this section, starting with some essential definition. What follows are important theorems that involved in this model. The construction of Nagai's GRA model is briefly described. More details are referred to the works of Yamaguchi et al. [16]-[20].

Definition 1. Let the set $X$ be a vector space to apply grey relational analysis, and the vectors $x, y$ are elements of $X$. The inner product of $\mathrm{x}$ and $\mathrm{y}$ is defined as follows:

$$
\langle x, y\rangle=\|x\|_{\xi},\|y\|_{\xi} \cos \theta
$$

where $x, y \in R^{n}$,

$$
\begin{gathered}
x=\left(x_{1}, x_{2}, \cdots, x_{n}\right)^{T} \\
\|x\|_{\xi}=\sqrt[\xi]{\sum_{i=1}^{n} x_{i}^{\xi}}
\end{gathered}
$$

The $X$ is content with the vector space axiom. Eq. (1) is satisfied with the inner product axiom. Both axioms are in set theory [21].

Definition 2. The metric between two vectors $x, y$ with the distinguish coefficient $\xi$ is defined as follows:

$$
\|x-y\|_{\xi}=\sqrt[\xi]{\sum_{i=1}^{n}\left|x_{i}-y_{i}\right|^{\xi}}
$$

where $\xi \geq 1$.

Eq. (4) is well known as Minkowski distance [21] or $L_{p}$ norm [21]-[24]. The Euclidean distance is the special case of Eq. (4) at $\xi=2$, and city-block distance is also special case of Eq. (4) at $\xi=1$ [21].

Axiom 1. The $X$ is a norm space, as consisted with the following three properties.
1) $\|x\|_{\xi} \geq 0$
2) $\|\alpha x\|_{\xi}=|\alpha| \cdot\|x\|_{\xi}$, where $\alpha \in R$
3) $\|x+y\|_{\xi} \leq\|x\|_{\xi}+\|y\|_{\xi}$

The third property in $L_{p}$ norm has been proved mathematically. Also, it has been proved that the norm space $X$ is Banach space in $L_{p}$ norm.

There two different approach of metric calculations. One is the traditional GRA and the other is called "proposal GRA" in [19]. For the traditional GRA, metric is calculated at each attributes independent. On the other hand, proposal metric at two-dimensional space and $\xi=2$, is the $\zeta$ root of a sum of difference at all attributes, and $\left\|x_{1}-x_{2}\right\|_{\xi}=\left\|x_{2}-x_{1}\right\|_{\xi}$. Traditional GRA's metric is more suitable for time series data, while the proposal metric is capable to employ the data set without time series. In this paper, the proposal GRA is used.

Definition 3. The following two features that are able to extract from traditional GRA concept are describe as follows:

1) The metric between two sequences is calculated, and normalized the grey relational grade with distinguish coefficient.

2) Grey relational grade has the order relation of each sequence.

Definition 4. The $\Gamma$ is a grey relational space, such as $\Gamma \subset X \times X$. The current GRA is a process that transfers Banach space into the grey relational space, and is content with Def. 3. The former is described by

$$
f: X \rightarrow \Gamma
$$

Definition 5. The variables $x_{0}$ and $x_{i}$ are both n-dimensional vectors, such as $x_{0}, x_{i} \in X$, which is the replaced sequence in GRA. Note that $x_{0}$ is a reference vector, and $x_{i}$ is an inspected vector, where $i=1,2, \cdots, m$.

Definition 6. The grey relational grade $\gamma_{i j}$ is defined as a value obtained by grey relational analysis, which is given for the ordered pair $\left(X_{i}, X_{j}\right) \subset \Gamma$.

Definition 7. The localized grey relational grade $\gamma_{0 i}$ can be defined as follows:

$$
\gamma_{0 i}=\frac{\Delta_{\max }-\Delta_{0 i}}{\Delta_{\max }-\Delta_{\min }}
$$

where

$$
\begin{aligned}
& \Delta_{0 i}=\left\|x_{0}-x_{i}\right\|_{\xi} \\
& \Delta_{\text {max }}=\max _{\forall i}\left\{\Delta_{0 i}\right\} \\
& \Delta_{\text {min }}=\min _{\forall i}\left\{\Delta_{0 i}\right\}
\end{aligned}
$$

Theorem 1. The globalized grey relational grade $\gamma_{i j}$ can be represented as follows:

$$
\gamma_{i j}=1-\frac{\Delta_{i j}}{\Delta_{\max }}
$$

where $i, j=1,2, \cdots, m$.

$$
\begin{gathered}
\Delta_{i j}=\left\|x_{i}-x_{j}\right\|_{\xi} \\
\Delta_{\max }=\max _{\forall i} \max _{\forall j}\left\{\Delta_{i j}\right\} .
\end{gathered}
$$

Eq. (7) is equivalent Eq. (6), such as

$$
\gamma_{i j}=\frac{\Delta_{\max }-\Delta_{i j}}{\Delta_{\max }-\Delta_{\min }}
$$

In Eq. (8), $\Delta_{\min }=\Delta_{i i}=0$ because $\Delta_{i i}$ becomes an oneself metric at $i=j$. Hence, Eq. (8) is described as follows: 


$$
\gamma_{i j}=\frac{\Delta_{\max }-\Delta_{i j}}{\Delta_{\max }}=1-\frac{\Delta_{i j}}{\Delta_{\max }}
$$

Definition 8. In the current GRA model, the grey relational matrix $\Gamma$ is defined as the following,

$$
\Gamma=\left[\begin{array}{cccc}
\gamma_{11} & \gamma_{12} & \cdots & \gamma_{1 m} \\
\gamma_{21} & \gamma_{22} & \cdots & \gamma_{2 m} \\
\vdots & \vdots & \ddots & \vdots \\
\gamma_{m 1} & \gamma_{m 2} & \cdots & \gamma_{m m}
\end{array}\right]
$$

The current GRA have the following properties, and several differences from traditional GRA are existent [19]-[28].

Theorem 2. Localized grey relational grade has the following three properties:

1) Normality: $0 \leq \gamma_{0 i} \leq 1\left(\gamma_{0 i} \in[0,1]\right)$

2) Isolation: $\left\|x_{0}-x_{i}\right\|_{\xi}=\Delta_{\max } \Leftrightarrow \gamma_{0 i}=0$

Closeness:

3)

$$
\left\|x_{0}-x_{i}\right\|_{\xi}=\Delta_{\min } \Leftrightarrow \gamma_{0 i}=1
$$

Theorem 3. Globalized grey relational grade also has the following four properties:

1) Normality: $0 \leq \gamma_{i j} \leq 1\left(\gamma_{i j} \in[0,1]\right)$

2) Isolation: $\left\|x_{i}-x_{j}\right\|_{\xi}=\Delta_{\max } \Leftrightarrow \gamma_{i j}=0$

\section{Coincidence:}

3) i) $\gamma_{i j}=1$

$$
\text { ii) } x_{i}=x_{j} \Leftrightarrow \gamma_{i j}=1
$$

4) Symmetry: $\gamma_{i j}=\gamma_{j i}$

Theorem 4. Localized and Globalized grey relational grade have both the order relation " $\prec$ " which is similar to traditional GRA [22], as follows:

1) Reflexive: $x_{i} \prec x_{i}$

2) Anti-symmetrical law: $x_{i} \prec x_{j}, x_{j} \prec x_{i} \Rightarrow x_{i}=x_{j}$

3)

Transitive law: $x_{i} \prec x_{j}, x_{j} \prec x_{k} \Rightarrow x_{i} \prec x_{k}$

where $i \neq j \neq k$

Theorem 5. Grey relational matrix $\Gamma$ is a symmetric matrix, and every diagonal element turns out to be $\gamma_{i i}=1$.

\section{RESULTS AND DISCUSSIONS}

This study focuses on sample data of 207 students mentioned in the previous section. Based on overview of prior literature [21]-[27], we derive broader coverage of significant variables for pictorial patterns (S2P1 means the question of sub-step 2 in Problem 1).

Grey Relational Analysis. Sample data on the above-mentioned problems of MWTCPM are used to calculate Minkowski distance of $L_{p}$ norm. Grey relational grade (Gamma) values are computed using current GRA model in Table I and Table II. Problems in these tables are sorted by average values of gamma.
From Table I, we find that all students get full scores for Problems in "reception" part of MWTCPM. Students can aware the professional contexts that are related to mathematics.

TABLE I: GAMMA VALUES FOR PROBLEMS IN “RECEPTION” ABILITY

\begin{tabular}{ll}
\hline \hline Problems & Gamma \\
\hline S1P1 & 0 \\
S1P2 & 0 \\
S1P3 & 0 \\
S1P4 & 0 \\
S1P5 & 0 \\
S1P6 & 0 \\
S1P7 & 0 \\
S1P8 & 0 \\
S1P9 & 0 \\
S1P10 & 0 \\
\hline \hline
\end{tabular}

TABLE II: GAMMA VALUES FOR PROBLEMS IN “TRANSFORMATION AND PROBLEM SOLVING" ABILITY

\begin{tabular}{ll}
\hline \hline Problems & Gamma \\
\hline S2P7 & 0 \\
S2P6 & 0.1377 \\
S2P9 & 0.2187 \\
S2P4 & 0.2807 \\
S2P1 & 0.3866 \\
S2P8 & 0.3866 \\
S2P3 & 0.4298 \\
S2P2 & 0.6317 \\
S2P10 & 0.8472 \\
S2P5 & 1 \\
\hline \hline
\end{tabular}

From Table II, Problem 7 [problem concerned unemployment rate] proves most difficult among 10 problems, followed by Problem 6 [problem concerned sales profit], Problem 9 [problem concerned exchange rate of area units], and Problem 4 [problem concerned discount of ticket of high-speed real way]. The authors wish to discern why university students have difficulty with these formats of exchange patterns. Researchers might consider this as their research interest.

On the other hand, GRA analysis shows Problem 5 [problem concerned discount of restaurant] as easiest for students, followed by Problem 10 [problem concerned exchange rate of currency] Problem 2 [problem concerned buy one get one], and Problem 3 [problem concerned insurance rate]. The authors wish to learn why university students have no difficulties with these formats of profit. The diagram depicts smooth distribution of gamma values from 0 to 1 .

Fig. 1 graphs average values of gamma, in which each point represents a question studied.

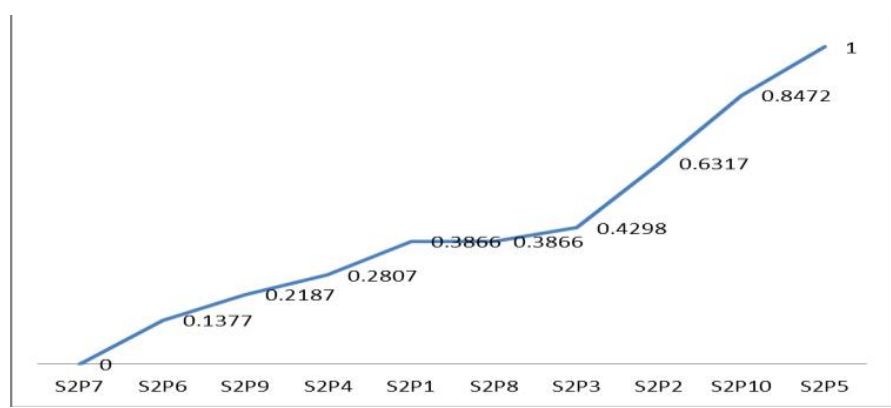

Fig. 1. Pattern of average grey relational grade (Gamma) for "transformation and problem solving" ability. 


\section{CONCLUSION}

Results of this study identified the easiest and most difficult concepts for university students; it is crucial to ferret out reasons for this outcome. The authors investigate why university students have difficulties in identifying the problem concerned rates (unemployment rate, sales profit, exchange rate of area units). One reason might be that the problem concerned rates are rarely shown in textbooks and/or professional mathematics. Researchers might consider it as their research interest and use Interpretive Structure Modeling (ISM) of Grey System, Fuzzy Measure [29]-[39], Fuzzy C-means [35], or the grey model $\operatorname{GM}(\mathrm{h}, \mathrm{N})$ [29]-[39], to analyze the reception, transformation and problem Communication ability of professional mathematics of university students. In the future, data on communication and evaluation can be analyzed by Fuzzy Measure, Fuzzy C-means, GRA, or Grey Model GM (h,N) also. Finally, we suggest that professors work the connection relative to professional mathematics, such that students apply math they learn and augment their long-life learning.

\section{REFERENCES}

[1] National Council of Teachers of Mathematics (NCTM), Curriculum and Evaluation Standards for School Mathematics, Reston, Virginia, NCTM 2000.

[2] Ministry of Education in Taiwan, "The nine-year-integration mathematics curriculum for primary and junior-high schools," 2008.

[3] M. J. Coles and W. D. Robinson, Teaching Thinking, Bristol: The Bristol Press, 1989.

[4] S. Krulik and J. A. Rudnick, Reasoning and Problem Solving: A Handbook for Elementary School Teachers, Needham Heights, Mass: Allyn and Bacon, 1993.

[5] S. Krulik and J. A. Rudnick, in Developing Mathematical Reasoning in Grades K-12, L. V. Stiff and F. R. Curcio, (Eds.), National Council of Teachers of Mathematics, 1999, pp. 138-145.

[6] P. G. O'Daffer and B. A. Thornquist, in Research Ideas for the Classroom: High School Mathematics, P. A. Wilson, (Ed.), New York: MacMillan Publishing, 1993, pp. 39-56.

[7] R. Audi, Practical Reasoning, London: Routledge, 1991.

[8] I. Murdoch The Sovereignty of Good London, Routledge/Ark, 1970

[9] C. Diamond, in Iris Murdoch and the Search for Human Goodness, M. Antonaccio and W. Schweiker, (Eds.), Chicago, University of Chicago Press, 1996.

[10] H. L. Ma, "2000 school year teacher normal education thesis bulleti," vol. 2, 2000, pp. 711-742.

[11] H. L. Ma, Chinese Journal of Science Education, vol. 9-4, 2001, pp. 375-399.

[12] H. L. Ma, Chinese Journal of Science Education, vol. 12-1, 2004, pp. 53-81.

[13] H L Ma, Chinese Journal of Science Education, vol. 15-4, 2007, pp. 387-416.

[14] H. L. Ma, D. B. Wu, Y. K. Cheng, and J. W. Chen, Advanced Materials Research, vol. 971-973, 2014, pp. 2705-2708

[15] H. L. Ma, C. I. Jen, S. H. Lin, and D. B. Wu, Advanced Materials Research, vol. 993, 2014, pp. 774-777.

[16] J. S. Jau, Master Thesis, Department of Automation Engineering and Institute of Mechatronoptic Systems, Chienkuo Technology University, Changhua, 2008.

[17] J. L. Deng, Grey System, China Oceans Press, China, 1988.

[18] M. Nagai and D. Yamaguchi, Elements on Grey System Theory and Applications, Kyoritsu-Shuppan, Tokyo, 2004.

[19] K. L. Wen, Grey Systems: Modeling and Prediction, Yang's Scientific Research Institute, Tucson, 2004.

[20] K. L. Wen, Apply MATLAB in Grey System Theory, CHWA Publisher, Taipei, Taiwan, 2006.

[21] D. Yamaguchi, G. D. Li, and M. Nagai, Journal of Grey System, vol. 10-13, 2007, pp. 169-182.

[22] K. L. Wen, C. W. Wang, and C. K. Yeh, in Proc. IEEE SMC 2007 Conference, Montreal, 2007, pp. 2271-2275.
[23] S. O. Chen, C. C. Huang, M. L .You, and C. Y. Kung, in Proc. Frontiers in the Convergence of Bioscience and Information Technologies, Korea ,2007.

[24] K. L. Wen, C. S. Choa et al., Culture Enterprise, Taipei, Taiwan, 2009.

[25] S. F. Liu and Y. Lin, Grey Information, London: Springer, 2005.

[26] M. L. You, C.W. Wang, and C. K. Yeh, Journal of Grey System, vol. 9, no. 1,2006 , pp. 57-64.

[27] D. Yamaguchi, T. Kobayashi et al., Japnanese Journal of Japan Society of Kansei Engineering, vol. 4, no. 2, 2004, pp. 101-106.

[28] D. Yamaguchi, G. D. Li, and M. Nagai, Journal of Grey System, vol. 8, no. 2, 2005, pp. 167-178.

[29] D. B. Wu and H. L. Ma, Journal of Grey System, vol. 12, no. 4, 2009, pp. 161-168.

[30] D. B. Wu and H. L.Ma, Applied Mechanics and Materials, vol. 44, no 47, 2011, pp. 3922-3926.

[31] H. C. Liu, C. C. Chen, D. B. Wu, and T. W. Sheu, WSEAS Transactions on Systems and Control, vol. 4, no. 8, 2009, pp. 359-368.

[32] H. C. Liu, D. B. Wu, Y. D. Jheng, and T. W. Sheu, WSEAS Transactions on Information Science and Applications, vol. 6, no. 6, 2009, pp. 1061-1070.

[33] H. C. Liu, D. B. Wu, J. M. Yih, and S. W. Liu, WSEAS Transactions on Biology and Biomedicine, vol. 4, no. 7, 2007, pp. 93-98.

[34] H. C. Liu, D. B. Wu, and H. L. Ma, WSEAS Transactions on Biology and Biomedicine, vol. 4, no. 7, 2007, pp. 99-102.

[35] H. C. Liu, J. M. Yih, W. C. Lin, and D. B. Wu, Journal of Multiple-Valued Logic and Soft Computing, vol. 15, no. 5-6, 2009, pp. 581-595.

[36] H. L. Ma, D. B. Wu, Y. K. Cheng, and J. W. Chen, Advanced Materials Research, vol. 971-973, 2014, pp. 2705-2708.

[37] H. L. Ma, C. J. Jen, S. H. Lin, and D. B. Wu, Advanced Materials Research, vol. 933, 2014, pp. 774-777.

[38] H. L. Ma, C. J. Jen, Y. K. Cheng, and D. B. Wu, Applied Mechanics and Materials, vol. 459, 2014, pp. 144-148.

[39] H. L. Ma, D. B. Wu, C. J. Jen, and Y. K. Cheng, Applied Mechanics and Materials, vol. 385-386, 2013, pp. 1893-1897.

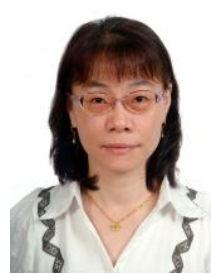

Hsiu-Lan Ma received her B. S. in mathematics from National Taiwan Normal University, Taipei, Taiwan in 1983, as well as M.S. and Ph.D. degrees in mathematics, University of Northern Colorado, Greeley, Colorado, USA in 1991 and 1994 respectively. Since 1996, she has been with the Department of Business Administration, Ling Tung University, where she is currently a professor. Her current interests include mathematics education, reasoning, problem solving, educational measurement, and web-based learning, etc.

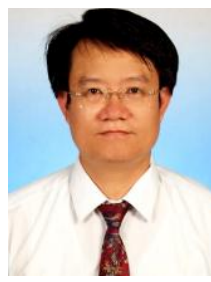

Der-Bang Wu received his B. S. and $M$. S. in mathematics from National Taiwan Normal University, Taipei, Taiwan in 1983 and 1985 . He earned his first doctoral degree (Ph.D.) from the Department of Mathematics, University of Northern Colorado, Greeley, Colorado, USA in 1994. Also, in order to earn his second doctoral degree, he is a doctoral student of Graduate Institute of Educational Measurement and Statistics, Taichung University since 2007. His studies focus in mathematics education, fuzzy c-mean, fuzzy measure, grey system, rough set, educational measurement, and e-learning, etc. Wu is a professor of the Department of Mathematics Education, Taichung University, Taichung, Taiwan. He was a teacher of Elementary School for 4 years (1979-1983) and work in Taichung University for 24 years (1985-2009), mainly engaged in the design and the development of mathematics textbooks, Research Instruments, van Hiele Geometrical Thinking, etc. Dr. Wu is a member of IEEE, Taiwan Association of Mathematics Education, Taiwan Association of Science Education, and International Group of Psychology of Mathematics Education, etc.

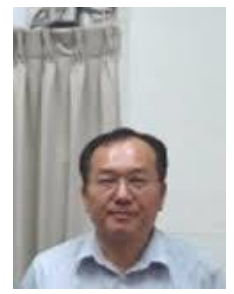

Yu-Kuang Cheng received his M. S. and doctora degree in English teaching from University Of Georgia, Georgia, U.S. He is an associated professor of the Department of English, Taichung University, Taichung, Taiwan. 